\title{
Computational assembly of a human Cytomegalovirus vaccine upon experimental epitope legacy
}

\author{
Monica J. Quinzo ${ }^{1}$, Esther M. Lafuente ${ }^{1}$, Pilar Zuluaga', Darren R. Flower ${ }^{2}$ and Pedro A. Reche ${ }^{1 *}$ (D) \\ From 2nd International Workshop on Computational Methods for the Immune System Function \\ Madrid, Spain. 3-6 December 2018
}

\begin{abstract}
Background: Human Cytomegalovirus (HCMV) is a ubiquitous herpesvirus affecting approximately $90 \%$ of the world population. HCMV causes disease in immunologically naive and immunosuppressed patients. The prevention, diagnosis and therapy of HCMV infection are thus crucial to public health. The availability of effective prophylactic and therapeutic treatments remain a significant challenge and no vaccine is currently available. Here, we sought to define an epitope-based vaccine against HCMV, eliciting B and T cell responses, from experimentally defined HCMVspecific epitopes.

Results: We selected 398 and 790 experimentally validated HCMV-specific B and T cell epitopes, respectively, from available epitope resources and apply a knowledge-based approach in combination with immunoinformatic predictions to ensemble a universal vaccine against HCMV. The T cell component consists of 6 CD8 and 6 CD4 T cell epitopes that are conserved among HCMV strains. All CD8 T cell epitopes were reported to induce cytotoxic activity, are derived from early expressed genes and are predicted to provide population protection coverage over 97\%. The CD4 T cell epitopes are derived from HCMV structural proteins and provide a population protection coverage over $92 \%$. The B cell component consists of just $3 \mathrm{~B}$ cell epitopes from the ectodomain of glycoproteins $\mathrm{L}$ and $\mathrm{H}$ that are highly flexible and exposed to the solvent.

Conclusions: We have defined a multiantigenic epitope vaccine ensemble against the HCMV that should elicit T and B cell responses in the entire population. Importantly, although we arrived to this epitope ensemble with the help of computational predictions, the actual epitopes are not predicted but are known to be immunogenic.
\end{abstract}

Keywords: HCMV, Epitopes, Vaccine, Prediction

\section{Background}

Human Cytomegalovirus (HCMV) seroprevalence is 50$90 \%$ in the adult population. HCMV can be transmitted via saliva, sexual contact, placental transfer, breastfeeding, blood transfusion, solid-organ transplantation or hematopoietic stem cell transplantation. The main risk factors for HCMV infection, reactivation and disease are: immune-naive state, immunosuppressive regimens, organ transplants and co-infection [1]. The prevalence of

\footnotetext{
* Correspondence: parecheg@med.ucm.es

${ }^{1}$ Faculty of Medicine, University Complutense of Madrid, Pza Ramon y Cajal, s/n, 28040 Madrid, Spain

Full list of author information is available at the end of the article
}

congenital HCMV infection has been estimated between $0.5-0.7 \%$ in the US, Canada and Western Europe and between 1 and 2\% in South America, Africa and Asia. Around $13 \%$ of infected infants are symptomatic with a wide range of phenotypes, including prematurity, intrauterine growth retardation, hepatomegaly, splenomegaly, thrombocytopenia, microcephaly, chorioretinitis, sensorineural hearing loss and focal neurologic deficits [2].

HCMV, or human herpesvirus 5, is a beta herpesvirus consisting of a $235 \mathrm{Kpb}$ double-stranded linear DNA core. HCMV genome is among the longest and most complex genomes of all human viruses, due to the diversity of wildtype strains in intrahost and interhost HCMV populations. 
The HCMV genome is translated in 3 overlapping phases (IE-immediate early: 0-2 h; E-early: < 24h; L-late: $>24$ h) giving rise to RNAs and proteins with a structural and/or a functional role in different stages of the viral cycle [3]. Davidson et al. [4] estimate that the wild-type HCMV genome carries 164-167 coding mRNAs accounting for one third of transcription, while 4 large non-coding RNAs account for $65.1 \%$.

Although HCMV can reside in both, myeloid and lymphoid lineages, monocytes are its primary target. HCMV reactivation and dissemination may occur after infected monocytes migrate into tissues and differentiate into macrophages since, unlike monocytes, they are permissive for viral gene expression [5]. Initial viral tethering occurs by engagement of glycoprotein $\mathrm{M} / \mathrm{N}$ to heparin proteoglycans, followed by binding of monocyte $\beta 1$ and $\beta 2$ integrins and epidermal growth factor receptor (EGFR). This binding activates downstream receptor signalling, which prompts viral entry and increases cellular motility, thus facilitating viral dissemination [6]. Once primary infection begins, there is a rapid innate response. Toll-like receptors (TLRs) interact with viral DNA starting the production of inflammatory cytokines, such as type I interferons (IFNs), which leads to an antiviral state and activates dendritic cells (DCs), macrophages and natural killer (NK) cells [7].

HCMV-specific adaptive immunity is required for long-lasting protective immunological memory, which prevents from reinfection, reactivation, uncontrolled replication and serious disease. Protection against HCMV is correlated with high frequencies of CD8 cytotoxic $\mathrm{T}$ lymphocytes (CTLs) specific for immediateearly 1 protein (IE-1) and $65 \mathrm{KDa}$ phosphoprotein (pp65) as well as type $1 \mathrm{CD} 4 \mathrm{~T}$ helper (Th1) cells specific for glycoprotein B (gB), TLR14 and UL16, which also exhibit cytotoxic activity [8-11]. Unlike T cells, B cells recognize solvent-exposed epitopes in target antigens. This recognition promotes $\mathrm{B}$ cell activation resulting in the secretion of antibodies (Abs) with the same specificity. Some protective anti-HCMV Abs have been shown to recognize envelope glycoprotein $\mathrm{B}(\mathrm{gB})$ and glycoprotein $\mathrm{H}(\mathrm{gH})$ [12].

Despite eliciting strong immune responses, HCMV has a large evasion armoury that is responsible for the resilience of the virus and its prevalence in the population. HCMV interferes with cytokine pathways, NK cell activation and antigen processing and presentation [13]. In addition, several studies point that numerous cycles of HCMV reactivation can lead to an early state of immune senescence, characterized by the decline of immune responsiveness, as well as the reduction in the levels of naive cells. This feature could be behind the association between chronic subclinical infection and long-term diseases such as atherosclerosis, chronic graft rejection, autoimmunity and certain neoplasias $[14,15]$.
Despite much effort, an effective treatment for HCMV disease remains a significant challenge. The most effective approach to prevent infection, transmission or reactivation in immune-naive or immunosuppressed individuals will be a multifunctional HCMV vaccine [16]. Currently, such a vaccine is not available. Vaccine development requires much effort, resource, and knowledge; yet the process can be facilitated greatly using immunoinformatics and related computational approaches [17-19]. Such approaches are particularly relevant for the design of epitope-based vaccines, which stand out for their safety and selectivity $[20,21]$. The design of epitope ensemble vaccines relies on sophisticated immunoinformatics tools, often based on machine learning, able to identify the majority of potential $\mathrm{T}$ and $\mathrm{B}$ cell epitopes from pathogen genomes [22, 23]. However, such predictions still require experimental validation, with only a few potential epitopes actually being immunogenic, and thus suitable for vaccine design [24].

Here, we designed multi-functional epitope-based vaccine for $\mathrm{HCMV}$ through an approach that combines legacy experimentation with immunoinformatic predictions [25-31]. The approach uses previously validated epitopes of proven immunogenicity obtained from public databases. A long list of experimentally-determined T-cell and B-cell epitopes is successively pruned by applying a series of sequence conservation, structural and immunological criteria. Subsequently, highly conserved epitopes meeting the required criteria are combined to minimise epitope number while retaining $90 \%$ or greater population protection coverage [25-31]. Our putative epitope ensemble vaccine should prove a viable starting point for the development of an effective vaccine against HCMV.

\section{Results}

\section{HCMV amino acid sequence variability}

Compared to other organisms, viruses have a high replication rate, displaying great sequence variability. This feature facilitates immune evasion and can hinder the development of vaccines providing protection to all strains. Such immune evasion can be better countered back with vaccines consisting of non-variable epitopes [20]. We analysed the amino acid sequence variability of HCMV proteins as a way of identifying non-variable epitopes (details in Methods). Briefly, we first clustered all HCMV protein sequences $(50,623)$ around a reference HCMV genome (NC_006273), obtaining representative protein clusters (162) for all but 9 of the ORFs included in the selected reference HCMV genome. We then produced multiple sequence alignments (MSAs) and subjected them to sequence variability analysis. We found that only 601 out of 62,196 residues had a variability $\mathrm{H} \geq 0.5$ (a site with $\mathrm{H} \leq 0.5$ is considered to be conserved). This extremely low variability is unexpected, 
even for a dsDNA virus, facilitating the selection of conserved epitopes for vaccine design. After these analyses, we selected only those epitopes that did not have any single residue with $\mathrm{H} \geq 0.5$.

\section{Selection of CD8 T cell epitopes}

We retrieved from IEDB (https://www.iedb.org/) 20 experimentally verified $\mathrm{HCMV}$-specific CD8 T cell epitopes from 499 available epitopes after the following search criteria: A) recognition by human subjects exposed to the virus and $\mathrm{B}$ ) induction of epitope specific CD8 T cells with killing activity over cells infected with HCMV. This type of selection guaranties that CD8 T cell epitopes are appropriately processed and presented by both, dendritic cells priming epitope-specific CD8 T cells and infected target cells. Of those, we discarded any peptide with variable residues and size out of the 9-11 residue-range as they are unlikely to bind class I human leukocytes antigen (HLA I) molecules. Thus, we retained 9 conserved CD8 $\mathrm{T}$ cell epitopes with a size between 9 and 11 residues that were subjected to HLA I binding predictions and population protection coverage (PPC), analyses (details in Methods). We found that just a single epitope (QYDPVAALF) could reach a PPC that is at the least of $66.71 \%$ (Table 1). We computed PPCs for 5 distinct ethnic groups in the USA populations and thus the minimum PPC is that reached in the group with the lowest coverage (details in Methods). The combined minimum PPC of all the peptides is $92.99 \%$ while the PPC for each ethnic group is: $99.76 \%$ for Blacks, 96.16\% for Caucasians, 98.18\% for Hispanics, 92.99\% for Native North Americans and 99.96 for Asians. The average PPC for the USA population is $97.41 \%$ and it can be reached by the combination of 6 epitopes: QYDPVAALF, NLVPMVATV, TTVYPPSSTAK, HERNGFTVL, QTVTST PVQGR, TPRVTGGGAM.

\section{Selection of CD4 T cell epitopes}

We obtained from IEDB (https://www.iedb.org/) 291 experimentally validated HCMV-specific CD4 T cell epitopes recognized by humans exposed to the HCMV. Of those, we selected 91 epitopes belonging to structural proteins for size and conservation analysis. Thus, we identified 77 conserved epitopes with a size between 9 and 21 amino acids, the usual length of peptides restricted by class II HLA (HLA II) molecules. These 77 epitopes belonged to pp65 (UL83) and gB (UL55). No conserved epitopes were identified in other structural proteins. Although these 77 epitope peptides were unique, some were largely overlapping. Therefore, we applied a clustering-based procedure (details in Methods) to identify shared epitopes defined by overlapping peptides. Thus, we proceeded with $37 \mathrm{CD} 4 \mathrm{~T}$ cell epitopes, 15 derived upon clusters, for HLA II binding and PPC analyses. In Table 2 we only report epitopes with $\mathrm{PPC} \geq 10 \%$. The maximum PPC obtained with all peptides was $92.49 \%$. However, we found that only 6 epitopes from the $65 \mathrm{KDa}$ phosphoprotein were necessary to achieve the same PPC: SIYVYALPLKMLNIP, KLFMHVTLGSDVEEDLT MTR, YQEFFWDANDIYRIF, LPLKMLNIPSINVHH, CSMENTRATKMQVIG and AGILARNLVPMVATV.

\section{Selection of B cell epitopes}

We found 398 experimentally validated HCMV-specific unique linear B cell epitopes generated during a natural infection. Of those, we focused on conserved epitopes mapping onto the ectodomain of envelope antigens so that they could induce protective Abs recognizing viral particles. Thus, we found 99 epitopes located in the ectodomains of glycoprotein H (UL75), glycoprotein L (UL115), glycoprotein B (UL55), glycoprotein M (UL100), glycoprotein UL4 (UL4), glycoprotein UL1 (UL1), TLR10 (IRL10) and TRL12 (IRL12). We clustered these epitopes

Table 1 HLA I binding profiles of conserved and experimentally verified HCMV-specific CTL epitopes

\begin{tabular}{|c|c|c|c|c|c|}
\hline Epitope & $\begin{array}{l}\text { Antigen } \\
\text { gene }\end{array}$ & $\begin{array}{l}\text { Antigen } \\
\text { accession }\end{array}$ & ${ }^{a}$ HLA I restriction & ${ }^{\mathrm{b}}$ Extended HLA I restriction & $\begin{array}{l}\text { C } \\
\text { PPC } \\
(\%)\end{array}$ \\
\hline QYDPVAALF & UL83 & Q6SW59 & $A^{*} 24: 02, A^{*} 01: 01, C^{*} 04: 01$ & $\begin{array}{l}A^{*}: 01: 01, A^{*} 24: 02, B^{*} 15: 16, B^{*} 38: 01, B^{*} 39011, B^{*} 3909, \\
B^{*} 5801, C^{*} 04: 01, C^{*} 07: 02\end{array}$ & 66.71 \\
\hline NLVPMVATV & UL83 & Q6SW59 & $\begin{array}{l}A^{*} 02: 01, A^{*} 02: 02, A^{*} 02: 03, A^{*} 02: 06, A^{*} 02: 11 \\
A^{*} 02: 12, A^{*} 02: 16, A^{*} 02: 19, A^{*} 24: 02, A^{*} 69: 01 \\
A^{*} 03, B^{*} 07: 02\end{array}$ & $\begin{array}{l}A^{*} 02: 01, A^{*} 02: 02, A^{*} 02: 03, A^{*} 02: 05, A^{*} 02: 06, A^{*} 02: 09, A^{*} 02: \\
14, A^{*} 68: 02, A^{*} 02: 12, A^{*} 02: 16, A^{*} 02: 19, A^{*} 02: 11, A^{*} 69: 01, \\
A^{*} 24: 02, B^{*} 07: 02\end{array}$ & 58.98 \\
\hline TTVYPPSSTAK & UL32 & Q6SW99 & $A^{*} 03: 01$ & $A^{*} 0301, B^{*} 15: 02, B^{*} 15: 08, C^{*} 07: 02$ & 34.47 \\
\hline HERNGFTVL & UL83 & Q6SW59 & $B^{*} 40: 01, B^{*} 40: 02, B^{*} 60$ & $A^{*} 2902, B^{*} 0702, B^{*} 1510, B^{*} 40: 01, B^{*} 40: 02$ & 24.21 \\
\hline TPRVTGGGAM & UL83 & Q6SW59 & $A^{*} 02: 01, B^{*} 07: 02$ & $A^{*} 02: 01, B^{*} 07: 02, B^{*} 55: 02, B^{*} 27: 06$ & 9.79 \\
\hline CEDVPSGKL & UL83 & Q6SW59 & $B^{*} 40: 01, A^{*} 03, B^{*} 60$ & $B^{*} 38: 01, B^{*} 40: 01$ & 2.78 \\
\hline QTVTSTPVQGR & UL32 & Q6SW99 & $A^{*} 68: 01$ & $A^{*} 33: 01, A^{*} 68: 01$ & 1.12 \\
\hline AELEGWWQPA & UL83 & Q6SW59 & $B^{*} 40: 06$ & $A^{*} 02: 09, B^{*} 40: 06$ & 0 \\
\hline
\end{tabular}

${ }^{a}$ Experimental restriction found in IEDB; ${ }^{b}$ Experimental plus predicted HLA I restriction/binding (details in Methods); ${ }^{\mathrm{c}}$ PPC was computed independently for 5 ethnic groups in the USA population using EPISOPT [27] and here we report the lowest PPC value 
Table 2 Predicted HLA II binding profile of conserved and experimentally verified HCMV-specific CD4 T cell epitopes

\begin{tabular}{|c|c|c|c|c|c|}
\hline Epitope & $\begin{array}{l}\text { Antigen } \\
\text { gene }\end{array}$ & $\begin{array}{l}\text { Antigen } \\
\text { accession }\end{array}$ & ${ }^{a}$ HLA I| restriction & ${ }^{\mathrm{b}}$ Extended HLA I| restriction & $\begin{array}{l}{ }^{c} P P C \\
(\%)\end{array}$ \\
\hline SIYYYALPLKMLNIP & UL83 & Q6SW59 & $\mathrm{DRB} 1 * 07, \mathrm{DRB} 1 * 15: 01$ & $\begin{array}{l}\text { DRB1*01:01, DRB1*03:01, DRB1*07:01, DRB1*11:01, } \\
\text { DRB1*15:01 }\end{array}$ & 63.95 \\
\hline KLFMHVTLGSDVEEDLTMTR & UL83 & Q6SW59 & $\mathrm{DRB} 1 * 03$ & $\begin{array}{l}\text { DRB1*03:01, DRB1*04:05, DQA1*05:01/ DQB1*02:01, } \\
\text { DQA 1*05:01/ DQB1*03:01 }\end{array}$ & 48.03 \\
\hline LPVADAVIHASGKQMWQARL & UL83 & Q6SW59 & $\mathrm{DRB} 1 * 03$ & $\begin{array}{l}\text { DRB1*01:01, DQA1*05:01/ DQB1*02:01, DQA1*05:01/ } \\
\text { DQB1*03:01 }\end{array}$ & 42.11 \\
\hline GPISGHVLKAVFSRG & UL83 & Q6SW59 & HLA class II & $\begin{array}{l}\text { DRB1*01:01, DQA } 1 * 05: 01 / \mathrm{DQB} 1 * 02: 01, \mathrm{DQA} 1 * 05: 01 / \\
\mathrm{DQB} 1 * 03: 01\end{array}$ & 42.11 \\
\hline CSMENTRATKMQVIG & UL83 & Q6SW59 & HLA class II & DRB1*07:01, DQA1*01:02/ DQB1*06:02 & 37.99 \\
\hline FTSHEHFGLLCPKSI & UL83 & Q6SW59 & HLA class II & DRB1*01:01, DRB1*07:01, DRB1*09:01, DRB5*01:01 & 34.02 \\
\hline YQEFFWDANDIYRIF & UL83 & Q6SW59 & $\mathrm{DRB} 1 * 01$ & DRB1*04:01, DRB3*01:01, DQA1*01:01/ DQB1*05:01 & 27.69 \\
\hline RNGFTVLCPKNMIIK & UL83 & Q6SW59 & HLA-DR & DRB1*01:01, DRB1*09:01, DRB1*11:01 & 27.09 \\
\hline RLLQTGIHVRVSQPS & UL83 & Q6SW59 & $\begin{array}{l}\text { DRB1*15, DQA1*01:02/ } \\
\text { DQB1*06:02 }\end{array}$ & $\mathrm{DQA} 1 * 01: 02 / \mathrm{DQB} 1 * 06: 02$ & 24.17 \\
\hline LRQYDPVAALFFFDI & UL83 & Q6SW59 & $\mathrm{DRB} 1 * 07$ & DRB1*07:01, DRB1*09:01, & 24.01 \\
\hline LPLKMLNIPSINVHH & UL83 & Q6SW59 & $\mathrm{DRB} 1 * 03$ & DRB1*01:01, DRB1*04:04, DRB1*09:01 & 21.87 \\
\hline QNLKYQEFFWDANDI & UL83 & Q6SW59 & HLA class II & DRB5*01:01, DQA1*01:01/ DQB1*05:01 & 18.56 \\
\hline EPDVYYTSAFVFPTK & UL83 & Q6SW59 & $\mathrm{DRB} 1 * 07$ & DRB $1 * 15: 01$ & 18.41 \\
\hline MLDVAFTSHEHFGLL & UL83 & Q6SW59 & HLA class II & DRB1*07:01 & 18.23 \\
\hline SDVEEDLTMTRNPQP & UL83 & Q6SW59 & $\mathrm{DRB} 1^{*} 03$ & DRB1*03:01 & 17.84 \\
\hline AGILARNLVPMVATV & UL83 & Q6SW59 & DRB1*11, DRB3*02:02 & DRB1*01:01, DRB3*02:02 & 11.53 \\
\hline EHPTFTSQYRIQGKL & UL83 & Q6SW59 & DRB1*11:01 & DRB1*11:01 & 10.54 \\
\hline TSQYRIQGKLEYRHT & UL83 & Q6SW59 & $\mathrm{DRB} 1 * 04, \mathrm{DRB} 1 * 13$ & DRB1*11:01 & 10.54 \\
\hline
\end{tabular}

${ }^{a}$ Experimental restriction found in IEDB; ${ }^{b}$ Experimental plus predicted HLA II restriction/binding (details in Methods); ${ }^{c}$ PPC was computed for 21 different ethnicities around the world

to identify common overlapping epitopes, finding only two epitopes from 2 sets of 4 and 7 overlapping epitopes (see Methods). All remaining 90 epitopes were fragmented into 9mers overlapping 8 amino acids, sought for conservation and clustered to identify the longest conserved fragment. Thus, we identified 15 conserved epitopes for which we computed their flexibility and accessibility (Table 3 ).

Since only one epitope (AFHLLLNTYGR) had a flexibility $\geq 1.0$ and an accessibility $\geq 48 \%$, determining their location in highly flexible and solvent-exposed regions [25], we sought for potential B cell epitopes from available crystal structures of HCMV envelope proteins (details in Methods) predicting $2 \mathrm{~B}$ cell epitopes, one in the ectodomains of the $\mathrm{gH}$ and another one in the ectodomain of the gL, that were also conserved (Table 4).

\section{Discussion}

There have been considerable efforts to develop a vaccine against HCMV, ranging from using attenuated viruses to various viral subunits [16]. However, there is currently no effective vaccine against HCMV. Subunit vaccines based on $\mathrm{gB}$ have shown $50 \%$ efficacy in preventing primary infection in young mothers and transplantation recipients, but they cannot prevent successive infections nor do they produce long-term protection [32,33]. Live recombinant vaccines based on replication-deficient viral vectors (e.g. poxvirus, adenovirus) encoding multiple $\mathrm{HCMV}$-specific epitopes have also been tested but they were poorly immunogenic and only after long periods of stimulation and expansion [34]. In this context, we designed a multi-functional epitope-based vaccine against the HCMV.

The main advantage of the epitope-based formulations is their exquisite selectivity as well as the possibility of inducing immune responses to subdominant epitopes and to various antigens at the same time. Moreover, they have been proposed to be safer than traditional vaccines $[20,35]$. Developing epitope-based vaccines is bound to the need to identify pathogen-specific epitopes within the relevant antigens, which, in spite of the available epitope prediction methods, is only achieved after laborious and costly experiments [22]. CD8 T cell epitope prediction methods are widely regarded as the most accurate and yet only $10 \%$ of predicted $\mathrm{T}$ cell epitopes are found to be immunogenic [36]. To bypass this problem, we formulated an epitope vaccine ensemble for HCMV through a computerassisted approach that feeds on previously identified 
Table 3 Conserved and experimentally verified B cell epitopes from HCMV envelope proteins

\begin{tabular}{llllll}
\hline Epitope & Antigen gene & ${ }^{a}$ Accession number & ${ }^{b}$ PDB & ${ }^{c}$ Flexibility & ${ }^{d}$ Accessibility (\%) \\
\hline VSIDDDTPML & UL75 & Q6SW67 & 5VOB: A [238-247] & 0.131 & 25.37 \\
TNQYLIKGISYPVST & UL75 & Q65W67 & 5VOB: A [592-606] & 0.09 & 32.63 \\
AFHLLLNTYGR & UL75 & Q6SW67 & 5VOB: A [37-47] & 1.618 & 55.08 \\
IFTEHVLGFELVPPS & UL115 & F5HCH8 & 5VOB: B [173-187] & -0.275 & 11.71 \\
TANQNPSPPWSKLTYSKPH & UL130 & F5HCP3 & 5VOB: D [32-50] & 0.104 & 39.64 \\
WSTLTANQNPSPPWSKLTY & UL130 & F5HCP3 & 5VOB: D [28-46] & 0.138 & 33.37 \\
FTYDTLRGYINRALA & UL55 & F5HB53 & 5C6T: A [486-500] & -0.644 & 64.02 \\
LRGYINRALAQIAEA & UL55 & F5HB53 & 5C6T: A [491-505] & -0.645 & 68.14 \\
NRALAQIAEAWCVDQ & UL55 & F5HB53 & 5C6T: A [496-510] & -0.724 & 63.41 \\
QIAEAWCVDQRRTLE & UL55 & F5HB53 & 5C6T: A [526-540] & -1.187 & 56.29 \\
SAILSAIYNKPIAAR & UL55 & F5HB53 & 5C6T: A [521-535] & -1.241 & 40.75 \\
SKINPSAILSAIYNK & UL55 & F5HB53 & 5C6T: A [516-530] & -1.322 & 46.13 \\
VFKELSKINPSAILS & UL55 & F5HB53 & 5C6T: A [481-495] & -0.734 & 38.57 \\
YAQLQFTYDTLRGYI & UL55 & F5HB53 & - & 0.442 & 53.73 \\
NVTFRGLQNKTEDFL & UL4 & Q65WC6 & & 19.39
\end{tabular}

${ }^{a}$ Accession number from UniProtKB database. ${ }^{b}$ Tertiary structure of the antigen (PDB code) with epitope location in square brackets. ${ }^{c}$ Average flexibility $\left(F_{b}\right.$, Eq. 3 ) of epitope in arbitrary units. ${ }^{d}$ Average relative solvent-exposed accessibility of epitope in percentage $\left(A_{b}\right.$, Eq. 4). The epitopes AFHLLLNTYGR and WSTLTANQNPSPPWSKLTY, were part of the epitopes AASEALDPHAFHLLLNTYGR and SWSTLTANQNPSPPWSKLTY, respectively. Accessibility and flexibility of NVTFRGLQNKTEDFL was predicted upon the antigen amino acid sequence as it did not map onto any 3D-structure (details in Methods)

epitopes readily available in specialized databases [37-40]. Clearly, the main advantage of this approach is the saving of time and resources as it depends on experimentally-validated epitopes. We first applied this approach for human immunodeficiency -1 virus and hepatitis $C$ virus, considering only CD8 T cell epitope vaccines [27, 29], later extending this to influenza A virus considering also CD4 T cell epitopes [31] and more recently to Epstein-Bar virus including B cell epitopes [25]. The keystone of this approach is to select conserved epitopes that are likely to induce protective immune responses (Fig. 1). In the specific case of HCMV, we selected CD8 T cell epitopes that are processed and presented both by antigen presenting cells (APCs) and HCMV infected cells, mediate cytotoxic activity and are derived from early expressed antigens. Consequently, memory CD8 T cells elicited by these epitopes will detect and kill infected cells early on avoiding virus dissemination. For CD4 T cell epitopes, we focused on epitopes presented by APCs from structural proteins so that they will provide early and effective help. Similarly, we only considered B cell epitopes mapping onto the ectodomain of envelope proteins so that they can elicit Abs recognizing the entire virus and block infection.
The epitopes obtained from the initial selection steps were subjected to different analysis for vaccine inclusion. The final epitope ensemble vaccine that we propose consists of 6 CD8 $\mathrm{T}$ cell epitopes, $6 \mathrm{CD} 4 \mathrm{~T}$ cell epitopes and $3 \mathrm{~B}$ cell epitopes (See Table 5). Conserved $\mathrm{T}$ cell epitopes were included in the ensemble for their ability to be presented by multiple HLA molecules providing maximum PPC. Thus, the CD4 and CD8 T cell epitope components are predicted to elicit responses in at least $90 \%$ of the population, regardless of their ethnicity. This level of response assumes that epitopes shown to be immunogenic in a specific HLA context will be also immunogenic in all the other HLA contexts defined by their HLA binding profile. Likewise, it assumes that antigen processing and appropriated epitope release remain the same in any HLA context. There is considerable evidence for these assumptions $[19,29]$. However, since epitope-HLA binding profiles are predicted, they will need confirmation for further vaccine development.

Conserved B cell epitopes in epitope ensemble vaccine were selected after flexibility and accessibility criteria and included one experimental epitope on $\mathrm{gH}$ and 2 predicted epitopes, one on gH and another on gL (Table 5).

Table 4 Predicted conserved B cell epitopes from HCMV envelope proteins

\begin{tabular}{llllll}
\hline Epitope & Antigen gene & ${ }^{a}$ Accession number & ${ }^{b}$ PDB & ${ }^{c}$ Flexibility & ${ }^{d}$ Accessibility (\%) \\
\hline TYNSSLRNS & UL75 & Q6SW67 & 5VOB: A [11-28] & 1.752 & 48.58 \\
TPEAANSVLLD & UL115 & F5HCH8 & 5VOB: B [57-69] & 1.397 & 58.36 \\
\hline
\end{tabular}

${ }^{a}$ Accession number from UniProtKB database. ${ }^{b}$ Tertiary structure of the antigen (PDB code) with epitope location in square brackets. ${ }^{c}$ Average flexibility of epitope in arbitrary unit $\left(F_{b}\right.$, Eq. 3). ${ }^{d}$ Average solvent-exposed accessibility of epitope in percentage $\left(A_{b}\right.$, Eq. 4) 


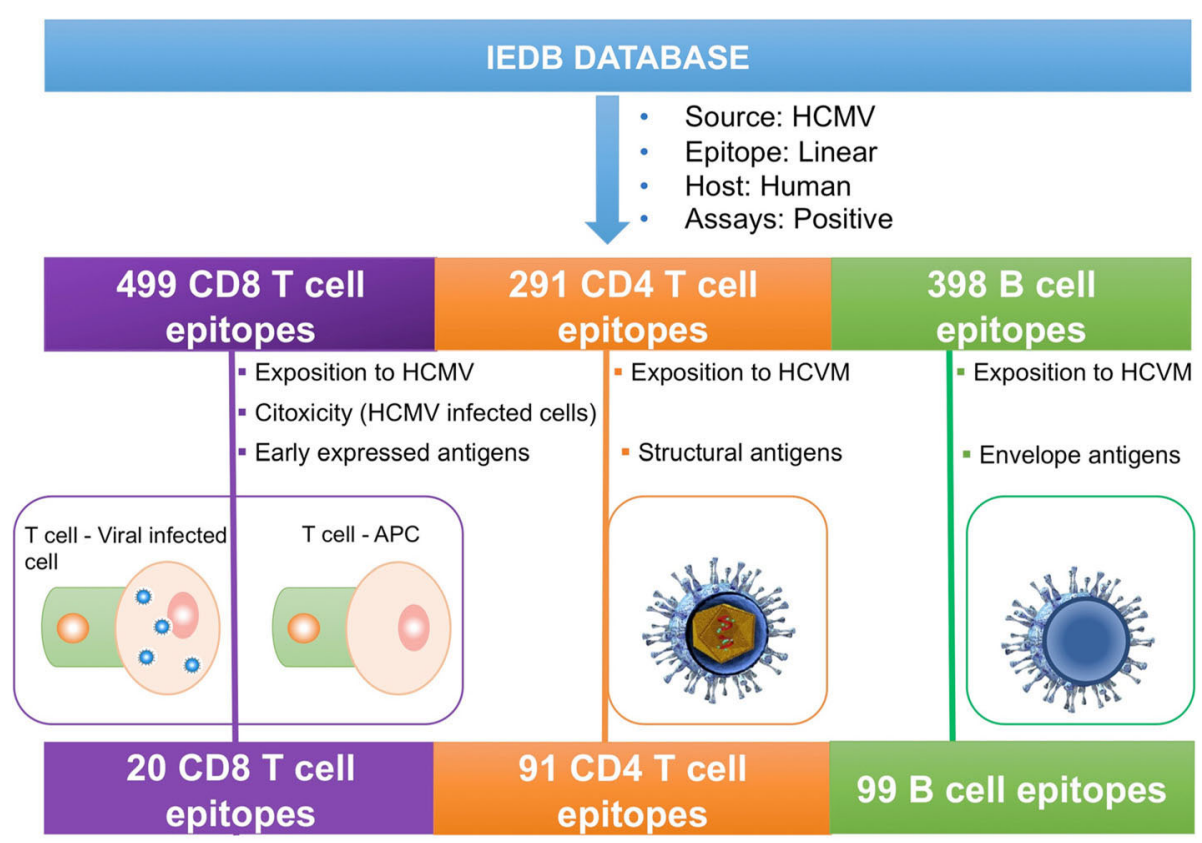

Knowledge-based Epitope selection for vaccine design

Fig. 1 Mapping of predicted (purple and blue) and experimentally defined (red) B cell epitopes on the tertiary structure of the $\mathrm{gH}$ and $\mathrm{gL}$ as part of the pentameric complex UL75/UL115/UL128/UL130/UL131A. B cell epitopes are respresented as sticks over a background of ribbons

The criteria of flexibility and accessibility that we applied were optimized to identify unstructured B cell epitopes lying in flexible and solvent exposed loop regions of the corresponding native antigens [25]. Consequently, these B-epitopes can be used as immunogens isolated from the antigen, e.g. as peptides, to induce the production of Abs that are likely cross-reactive with the native antigen [22].

All the epitopes in the proposed epitope ensemble are highly conserved to avoid or reduce immune evasion caused by viral genetic drift. Interestingly, we found that despite HCMV having very low sequence variability ( $1 \%$ of variable residues) only $40 \%$ of the selected $\mathrm{T}$ cell epitopes and 15\% of the selected B cell epitopes are conserved. These results indicate that sequence variability enables HCMV to escape the immune response, particularly the $\mathrm{Ab}$ response. They also highlight the crucial role of $\mathrm{T}$ cell responses in the control of HCMV in infected individuals.

Our epitope ensemble vaccine is multiantigenic, targeting 4 different HCMV proteins: pp65 (UL83), 150KDa phosphoprotein (pp150, UL32), envelope gL (UL115) and envelope gH (UL75). There are 2 antigens represented in the CD8 $\mathrm{T}$ cell epitope component (pp65 and pp150) and 2 antigens in B cell epitope component (gL and gH). However, CD4 T cell component only contains epitopes from the pp65. Arguably, it would have been better to include epitopes from some other antigens in the CD4 $\mathrm{T}$ cell component. However, the selected $\mathrm{CD} 4 \mathrm{~T}$ cell epitopes do provide the maximum
PPC and ought to offer effective help to both CD8 T cells and B cells.

Three of the targeted antigens (UL83, UL115 and UL75) have been included in other vaccines currently undergoing clinical trials, highlighting the importance of these antigens as components of a HCMV-specific vaccine. The viral protein pp65 (UL83) is delivered to infected cells as a virion component and rapidly moves to the nucleus where it antagonizes the cellular antiviral response through the NF-kB pathway [41]. The viral protein pp150 (UL32) associates with the nuclear viral capsids before DNA encapsidation and later protects nucleocapsids along secondary envelopment at the assembly compartment [42]. $\mathrm{gH}$ and gL are part of the $\mathrm{gH} / \mathrm{gL} / \mathrm{gO}$ trimeric complex and the $\mathrm{gH} / \mathrm{gL} / \mathrm{UL} 128 /$ UL130/UL131A pentameric complex which are important for viral entry into fibroblasts (trimeric complex) and epithelial and endothelial cells (pentameric complex) [43]. It has been shown that antibodies targeting gL/gH can hinder assembly of both complexes blocking HCMV entry into host cells [43]. Interestingly, the three B cell epitopes selected in this study are in regions of $\mathrm{gL}$ and $\mathrm{gH}$ interacting with proteins of the trimeric and pentameric complexes (Fig. 2). Thereby, we speculate that Abs elicited by these $3 \mathrm{~B}$ cell epitopes will block HCMV entry in fibroblasts and epithelial and endothelial cells. HCMV has additional proteins that are also important for entry in other cell types such as $\mathrm{gB}$ and the $\mathrm{gM} / \mathrm{gN}$ complex that are involved in HCMV infection of 
Table 5 Epitope ensemble vaccine for HCMV

\begin{tabular}{|c|c|c|c|c|c|}
\hline Epitope & Antigen gene & Antigen accession & BLAST hit human (\%) & BLAST hit HMP (\%) & PPC (\%) \\
\hline \multicolumn{6}{|c|}{ CD8 T cell epitope vaccine component } \\
\hline QYDPVAALF & UL83 & Q6SW59 & NP_060360.3 (66.67) & ETS98202.1 (78.00) & 66.71 \\
\hline NLVPMVATV & UL83 & Q6SW59 & AMD82163.1 (66.67) & KWZ77571.1 (78.00) & 58.98 \\
\hline TTVYPPSSTAK & UL32 & Q65W99 & CAC15059.1 (63.64) & OFS88048.1 (72.73) & 34.47 \\
\hline HERNGFTVL & UL83 & Q6SW59 & XP_016879611.1 (77.78) & EGG81521.1 (77.78) & 24.21 \\
\hline TPRVTGGGAM & UL83 & Q6SW59 & AGP01160.1 (70.00) & EJD64485.1 (80.00) & 9.79 \\
\hline QTVTSTPVQGR & UL32 & Q6SW99 & NP_005892.1 (63.64) & EHY57399.1 (82.00) & 1.12 \\
\hline \multicolumn{6}{|c|}{ CD4 T cell epitope vaccine component } \\
\hline SIYVYALPLKMLNIP & UL83 & Q6SW59 & XP_006724177.2 (46.67) & AEA20835.1 (73.33) & 63.95 \\
\hline KLFMHVTLGSDVEEDLTMTR & UL83 & Q6SW59 & EAX02867.1 (45.00) & ERJ00718.1 (45.00) & 48.03 \\
\hline YQEFFWDANDIYRIF & UL83 & Q6SW59 & EAW98902.1 (46.67) & EIJ68880.1 (60.00) & 27.69 \\
\hline LPLKMLNIPSINVHH & UL83 & Q6SW59 & EAX02055.1 (46.67) & EEU31012.1 (73.33) & 21.87 \\
\hline CSMENTRATKMQVIG & UL83 & Q6SW59 & EAW54200.1 (53.33) & EGL44246.1 (73.33) & 37.99 \\
\hline AGILARNLVPMVATV & UL83 & Q6SW59 & BAC11142.1 (46.67) & EFV13316.1 (73.33) & 11.53 \\
\hline \multicolumn{6}{|c|}{ B cell epitope vaccine component } \\
\hline AFHLLLNTYGR & UL75 & Q6SW67 & EAW77317.1 (63.64) & KXA44279.1 (63.64) & \\
\hline${ }^{*}$ TYNSSLRNS & UL75 & Q6SW67 & AHA56189.1 (78.00) & EFE23794.1 (90.00) & \\
\hline *TPEAANSVLLD & UL115 & $\mathrm{F} 5 \mathrm{HCH} 8$ & NP_001017403.1 (72.73) & OFU98142.1 (72.73) & \\
\hline
\end{tabular}

We show closest blast hits to human Proteins and Human Microbiome Proteins. The B cell epitope component contains one epitope validated experimentally and 2 predicted epitopes $(*)$. PPC of the entire CD8 and CD4 T cell component is 97.41 and $92.49 \%$

monocytes [43]. It would have been desirable to have these HCMV envelope proteins represented in the B cell epitope component of our vaccine. Unfortunately, we could not identify conserved B cell epitopes meeting our criteria of flexibility and accessibility in such proteins.

A potential adverse effect of vaccines is that of inducing immune responses cross-reactive with self-antigens. Thereby, we verified that none of the included epitopes matched exactly human proteins or human microbiome proteins. The sequence similarity of all epitopes with human proteins is less than $80 \%$; only two epitopes have a similarity over $80 \%$ with microbiome proteins. Since immune recognition is exquisitely specific, it can be disrupted by single amino acid mutation [44], and it is unlikely that the epitope ensemble proposed here will elicit harmful self-immune responses.

\section{Conclusions}

We have assembled a HCMV vaccine consisting of 6 CD8 T, 6 CD4 T and $3 \mathrm{~B}$ cell epitopes from 4 different HCMV antigens. The epitopes do not match self proteins, are conserved and all but $2 \mathrm{~B}$ cell epitopes are experimentally verified and reported to be recognized by humans exposed to HCMV. This epitope ensemble was built using a knowledge-based, computer assisted approach aimed at identifying epitopes that are likely to induce protective adaptive immune responses. Thus, the $\mathrm{T}$ cell epitopes are predicted to provide a PPC over $90 \%$ and include CD8 T cell epitopes mediating cytoxicity against HCMV infected cells. The B cell epitopes are all in highly flexible and accessible regions of the ectodomain of $\mathrm{gH}$ and $\mathrm{gL}$ proteins which makes them suitable for inducing Abs cross-reactive with the relevant native antigens. Moreover, they are proximally located to regions involved in the assembly of key complexes for viral entry. Thus, Abs induced by these epitopes could be neutralizing and block infection.

We have sought to identify optimal epitope components for making a protective HCMV vaccine, but there remains a long road ahead prior to deploying a preventive vaccine. Epitope peptides are known to be poorly immunogenic and the epitope ensemble will have to be contained within a formulation capable of inducing potent innate and adaptive immune responses. An attractive formulation will be to encapsulate the $\mathrm{T}$ cell epitopes along with appropriated adjuvant on liposome-based nanoparticles, displaying the B cell epitopes on the outer surface [45].

\section{Methods}

Collection of HCMV-specific immunogenic epitopes and 3D-structures of HCMV envelope proteins

Experimentally confirmed HCMV-specific epitopes were obtained from IEDB [46]. We only considered epitopes producing positive assays with humans as the host. In addition, we applied different search criteria to $\mathrm{B}$ and $\mathrm{T}$ cell epitopes. For B cell epitopes, we considered any 


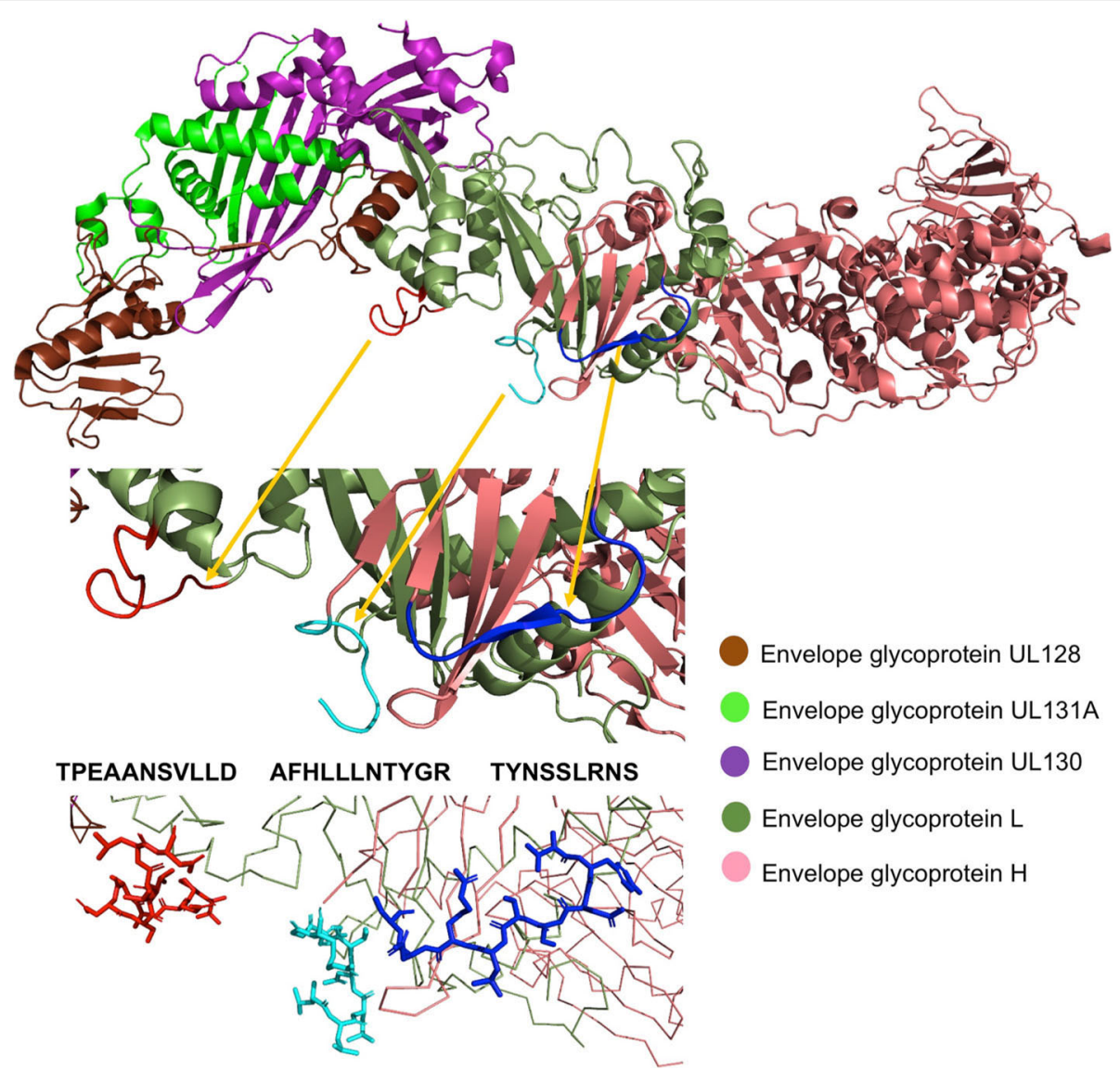

Fig. 2 Knowledge-based selection of experimental epitopes for HCMV vaccine design. Experimental epitopes were obtained form IEDB and selected to identify those that are more likely to induce protective immunity in humans. CD8 T cell epitopes were identified upon searches that guarantee that were processed and presented early by APCs (immunogen exposition) and by target cells (mediate cytotoxic activity of cells infected with HCMV). CD4 T cell epitopes were selected for being recognized by HCMV exposed subjects and belonging to structural proteins, so that they will provide early effective help. B cell epitopes were also selected for being recognized by HCMV exposed subjects and mapping onto the ectodomain of envelope proteins so that they can induce neutralizing antibodies

linear peptide from HCMV while we only considered HCMV-specific $\mathrm{T}$ cell epitopes that were elicited in humans exposed to the HCMV. In addition, for CD8 T cell epitopes, we restricted the selection to those that were reported to test positive on ${ }^{51} \mathrm{Cr}$ cytotoxic assays with cells infected with HCMV (relation between epitope and antigen is source organism).

\section{Multiple sequence alignment of HCMV proteins and generation of consensus proteins through sequence variability analysis}

We used CD-HIT [47] to cluster HCMV protein sequences $(50,623)$ - obtained from NCBI taxonomy database (TAX ID: 10359) [48] and including the open reading frames (ORFs) of a reference HCMV genome (NC_006273)-, using an identity threshold of $85 \%$. Subsequently, we selected those clusters containing reference sequences and produced multiple sequence alignments (MSA) using MUSCLE [49].

Sequence variability of the MSA was analysed per site/ position using the Shannon Entropy $(H)$ [50], as the variability metric (Eq. 1).

$$
H=-\sum_{i}^{M} P_{i} \log _{2}\left(P_{i}\right)
$$

where $P_{i}$ is the fraction of residues of amino acid type $i$ and $M$ is the number of amino acid types. $H$ ranges from 0 (only one amino acid type is present at that position) to 4.322 (every amino acid is equally represented in that position). Following these calculations, we masked in the reference HCMV proteome (NC_006273) any site with $H \geq 0.5$, thus generating consensus sequences. HCMV epitopes that matched entirely with the consensus HCMV sequences were retained for subsequent analysis. 


\section{Simplification of epitope datasets containing overlapping peptides}

We used CD-HIT [47] to identify clusters of overlapping peptide sequences in the CD4 and B cell epitope datasets. MSAs generated after the relevant clusters were processed so that overlapping epitopes were then represented by the common core defined by the MSA. For CD4 T cell epitopes, the common core was extended up to a length 15 residues when needed, adding relevant $\mathrm{N}$ - and/or $\mathrm{C}$ terminal residues. No common core longer than 15 residues was identified for overlapping CD4 T cell epitopes.

\section{Prediction of peptide HLA binding profiles and computation of population protection coverage}

We predicted binding of CD8 T cell epitopes to 55 HLA I molecules using EPISOPT (http://imed.med.ucm.es/Tools/ episopt.html) [27]. EPISOPT uses profile-motifs to predict peptide-MHC binding [51, 52] and considers peptides as HLA binders when their score is within the top $2 \%$ percentile. HLA I allele specific profile-motifs in EPISOPT only predict binding of 9mer peptides, which is the most common size of peptides found to bind HLA I molecules [53]. For longer peptides, HLA I binding profiles were obtained evaluating the binding of all 9mer peptides within the longer peptide. For CD4 T cell epitopes, we predicted peptide binding to a reference set of 27 HLA II molecules [54] with IEDB tools (http://tools.iedb.org/mhcii/). The reference set includes HLA II molecules belonging to HLA-DP, HLADQ and HLA-DR genes and a 5\% percentile rank was used to assess binding. As the prediction method, we selected "IEDB recommended". This method provides a consensus prediction which combines matrix and neural networkbased models, when the relevant predictors are available, otherwise returning predictions provided by NetMHCIIpan [55]. For peptides longer than 15 residues, predicted HLAII binding profiles corresponded to all 15-mers overlapping 14 amino acids contained in the longer peptide. Epitope population protection coverage (PPC) was computed with EPISOPT [27] for CD8 T cell epitopes and with the IEDB PPC tool for CD4 T cell epitopes (http://tools.iedb.org/ tools/population/iedb_input) [56]. EPISOPT computes the PPC for 5 distinct ethnic groups prevalent in North America (Black, Caucasian, Hispanic, Asian and Native North American), accounting for linkage disequilibrium between HLA I alleles [27], and identifies epitope ensembles reaching a determined PPC. The IEDB PPC tool does not consider linkage disequilibrium between HLA II alleles but does include allele frequency for 21 different ethnicities around the world [56].

\section{Computation of flexibility and accessibility of B cell epitopes}

The flexibility and accessibility of B cell epitopes was predicted using the relevant Protein Data Bank (PDB) files, when available, as described elsewhere [25]. Briefly, we computed normalized $\mathrm{C} \alpha$ B-factors, $Z_{B i}$ (Eq. 2), after the PDBs and used them as a measure of flexibility:

$$
Z_{B i}=\frac{\left(B_{i}-\mu_{B}\right)}{\partial_{B}}
$$

In Eq. $2, \mathrm{~B}_{\mathrm{i}}$ is the $\mathrm{B}$ factor of the $\mathrm{C} \alpha$ from residue $i$, obtained from relevant PDB, $\mu_{B}$ is the mean of $C \alpha$ B factors, and $\partial_{B}$ is the corresponding standard deviation. Likewise, we used NACCESS [57] to compute residue relative solvent accessibility (RSA) from the relevant PDBs.

Subsequently, we used Eq. 3 and 4 to compute an average flexibility $\left(F_{b}\right)$ and accessibility $\left(A_{b}\right)$, respectively, for each B cell epitope.

$$
\begin{aligned}
F_{b} & =\frac{\sum_{i=1}^{i=n} Z_{B i}}{n} \\
A_{b} & =\frac{\sum_{i=1}^{i=n} R S A_{i}}{n}
\end{aligned}
$$

where $\mathrm{n}$ is the total number of residues encompassed by the B cell epitope.

For B cell epitope sequences in antigens without solved tertiary structure, we predicted residue RSA and normalized B values with NetSurfP [58] and profBval [59], respectively, using as input the entire antigen sequence. Subsequently, we computed $F_{\mathrm{b}}$ and $A_{\mathrm{b}}$ values with predicted $\mathrm{B}$ and RSA values of the relevant residues (Eq. 3 and 4). We also used Eq. 3 and 4 for de novo prediction of potential B cell epitopes within selected HCMV antigens of known tertiary structures. Specifically, we considered as B cell epitopes those fragments consisting of 9 or more consecutive residues with a $F_{\mathrm{b}} \geq$ 1.0 and an $A_{b} \geq 48 \%$. Peptides fitting these structural criteria are found to be located in highly flexible and solvent-exposed regions of the antigen [25].

\section{Other procedures}

We used BLAST searches [60] against the PDB database subset at NCBI to map B cell epitopes onto 3D-structures and retrieve the relevant PDBs. We also used BLAST searches to determine sequence identity between epitopes and human or human microbiome proteins as described elsewhere [25]. For these searches, we used the NCBI non-redundant (NR) collection of human proteins and the human microbiome protein sequences obtained from the NIH Human Microbiome Project at NCBI (https://www.ncbi.nlm.nih.gov/bioproject/43021). We visualized 3D-structures and produced molecular renderings using the PyMOL Molecular Graphics System, Version 1.2r3pre, Schrödinger, LLC. 


\section{Abbreviations}

Ab: Antibody; gB: Glycoprotein B; gH: Glycoprotein H; gH: Glycoprotein M gL: Glycoprotein L; HCMV: Human Cytomegalovirus; HLA: Human Leukocyte Antigen; MHC: Major Histocompatibility Complex; pp65: 65 KDa phosphoprotein; RSA: Relative Solvent Accessibility

\section{Acknowledgements}

We wish to thank the Spanish Department of Science at MINECO for continuous support of the research of the Immunomedicine group through grants SAF2006:07879, SAF2009:08301 \& BIO2014:54164-R to PAR.

\section{About this supplement}

This article has been published as part of BMC Bioinformatics Volume 20 Supplement 6, 2019: Towards computational modeling on immune system function. The full contents of the supplement are available online at https:// bmcbioinformatics.biomedcentral.com/articles/supplements/volume-20supplement-6.

\section{Authors' contributions}

Conceptualization: EML, DRF \& PAR.; Methodology: MJQ, PZ \& PAR; Investigation: MJQ, EML, PZ, \& PAR. Writing-Original Draft: MJQ \& PAR; Final Writing \& Editing: DRF, EML \& PAR. All authors have read and approved the final manuscript.

\section{Funding}

The work was supported by grant BIO2014:54164-R from Spanish Department of Science. Publication costs funded by Complemento II-CM network (S2017/BMD-3673)

\section{Availability of data and materials}

Epitope datasets analyzed in this study were obtained and are available at the IEDB resource (http://www.iedb.org/) and from the corresponding author on reasonable request.

\section{Ethics approval and consent to participate}

Not applicable.

\section{Consent for publication}

Not applicable.

\section{Competing interests}

The authors declare that they have no competing interests.

\section{Author details}

${ }^{1}$ Faculty of Medicine, University Complutense of Madrid, Pza Ramon y Cajal, s/n, 28040 Madrid, Spain. ${ }^{2}$ School of Life and Health Sciences, Aston University, Aston Triangle, Birmingham B4 7ET, UK.

Received: 12 July 2019 Accepted: 23 August 2019

Published: 12 December 2019

\section{References}

1. Krause PR, Bialek SR, Boppana SB, Griffiths PD, Laughlin CA, Ljungman P, Mocarski ES, Pass RF, Read JS, Schleiss MR, et al. Priorities for CMV vaccine development. Vaccine. 2013:32(1):4-10.

2. Kenneson A, Cannon MJ. Review and meta-analysis of the epidemiology of congenital cytomegalovirus (CMV) infection. Rev Med Virol. 2007;17(4):253-76.

3. Wathen MW, Stinski MF. Temporal patterns of human cytomegalovirus transcription: mappings the viral RNAs synthesized at immediate early, early, and late times after infection. J Virol. 1982:41:462-77.

4. Davidson AJ, Dolan A, Akter P, Addison C, Dargan DJ, Alcendor DJ, McGeoch DJ, Hayward GS. The human cytomegalovirus genome revisited: comparison with the chimpanzee cytomegalovirus genome. J Gen Virol. 2003:84:17-28

5. Taylor-wiedeman J, Sissons JG, Borysiewicz LK, Sinclair JH. Monocytes are a major site of persistence of human cytoegalovirus in peripheral blood mononuclear cells. J Gen Virol. 1991;72(9):2059-64.

6. Chang G, Nogalski MT, Yurochko AD. Activation of EGFR on monocytes is required for human cytomegalovirus entry and mediates cellular motility. Proc Natl Acad Sci U S A. 2009;106:22369-74.
7. Juckem LK, Boehme KW, Feire AL, Compton T. Differential initiation of innate immune responses induced by human cytomegalovirus entry into fibroblast cells. J Immunol. 2008;180:4965-77.

8. Bunde T, Kirchner A, Hoffmeister B, Habedank D, Hetzer R, Cherepnev G, Proesch S, Reinke P, Volk HD, Lehmkuhl H, et al. Protection from cytomegalovirus after transplantation is correlated with immediate early 1 specific CD8 T cells. J Exp Med. 2005;201(7):1031-6. https://doi.org/10.1084/ jem.20042384 Epub 20042005 Mar 20042328.

9. Crough T, Fazou C, Weiss J, Campbell S, Davenport MP, Bell SC, Galbraith A, McNeil K, Khanna R. Symptomatic and asymptomatic viral recrudescence in solid-organ transplant recipients and its relationship with the antigenspecific CD8(+) T-cell response. J Virol. 2007;81(20):11538-42.

10. Hegde NR, Dunn C, Lewinsohn DM, Jarvis MA, Nelson JA, Johnson DC. Endogenous human cytomegalovirus $\mathrm{gB}$ is presented efficiently by $\mathrm{MHC}$ class II molecules to CD4+ CTL. J Exp Med. 2005;202(8):1109-19. https://doi. org/10.1084/jem.20050162 Epub 20052005 Oct 20050110

11. Casazza JP, Betts MR, Price DA, Precopio ML, Ruff LE, Brenchley JM, Hill BJ, Roederer M, Douek DC, Koup RA. Acquisition of direct antiviral effector functions by CMV-specific CD4+ T lymphocytes with cellular maturation. J Exp Med. 2006;203(13):2865-77.

12. Gamadia LE, Remmerswaal EB, Weel JF, Bemelman F, van Lier RA, Ten Berge IJ. Primary immune responses to human CMV: a critical role for IFN-gammaproducing CD4+ T cells in protection against CMV disease. Blood. 2003; 101(7):2686-92

13. Abbas AK, Lichtman AH, Shiv P. Cellular and molecular immunology, 8 edn: Elsevier; 2015.

14. Khan N, Hislop A, Gudgeon N, Cobbold M, Khann R, Nayak L, Rickinson AB. Herpesvirus-specific CD8 T cell immunity in old age cytomegalovirs impairs the response to a coresident EBV infection. J Immunol. 2004;173:7481-9.

15. Pourgheysari B, Khan N, Best D, Bruton R, Nayak L, Moss PA. The cytomegalovirus-specific CD4 ${ }^{+}$T-cell response expands with age and markedly alters the CD4 ${ }^{+}$T-cell repertoire. J Virol. 2007:81(14):7759-65.

16. Schleiss MR. Cytomegalovirus vaccines under clinical development. J Virus Erad. 2016:2(4):198-207.

17. Gomez-Perosanz M, Russo G, Sanchez-Trincado J, Pennisi M, Reche P, Shepherd A, Pappalardo F. Computational immunogenetics. In: Encyclopedia of bioinformatics and computational biology, vol. 2. Amsterdam: Elsevier; 2018. p. 906-30.

18. Vivona S, Gardy JL, Ramachandran S, Brinkman FS, Raghava GP, Flower DR, Filippini F. Computer-aided biotechnology: from immuno-informatics to reverse vaccinology. Trends Biotechnol. 2008;26(4):190-200. https://doi.org/1 0.1016/j.tibtech.2007.12.1006 Epub 2008 Feb 1021.

19. Sette A, Rappuoli R. Reverse vaccinology: developing vaccines in the era of genomics. Immunity. 2010;33(4):530-41. https://doi.org/10.1016/j.immuni.2 010.09.1017.

20. Sette A, Fikes J. Epitope-based vaccines: an update on epitope identification, vaccine design and delivery. Curr Opin Immunol. 2003;15(4):461-70.

21. Toussaint NC, Kohlbacher O. Towards in silico design of epitope-based vaccines. Expert Opin Drug Discov. 2009:4(10):1047-60. https://doi.org/1 0.1517/17460440903242283 Epub 17460440903242009 Aug 17460440903242228

22. Sanchez-Trincado JL, Gomez-Perosanz M, Reche PA. Fundamentals and methods for T- and B-cell epitope prediction. J Immunol Res. 2017:2017: 2680160.

23. Dhanda SK, Usmani SS, Agrawal P, Nagpal G, Gautam A, Raghava GPS. Novel in silico tools for designing peptide-based subunit vaccines and immunotherapeutics. Brief Bioinform. 2017;18(3):467-78. https://doi.org/10.1 093/bib/bbw1025

24. Lehmann PV, Suwansaard M, Zhang T, Roen DR, Kirchenbaum GA, Karulin AY, Lehmann A, Reche PA. Comprehensive Evaluation of the Expressed CD8+ T Cell Epitope Space Using High-Throughput Epitope Mapping. Front Immunol. 2019:10:655. https://doi.org/10.3389/fimmu.2019.00655 eCollection 02019.

25. Alonso-Padilla J, Lafuente EM, Reche PA. Computer-aided design of an epitope-based vaccine against epstein-barr virus. J Immunol Res. 2017;2017: 9363750.

26. Damfo SA, Reche P, Gatherer D, Flower DR. In silico design of knowledgebased Plasmodium falciparum epitope ensemble vaccines. J Mol Graph Model. 2017:78:195-205

27. Molero-Abraham M, Lafuente EM, Flower DR, Reche PA. Selection of conserved epitopes from hepatitis $C$ virus for pan-population stimulation of T-cell responses. Clin Dev Immunol. 2013;2013:601943. 
28. Murphy D, Reche P, Flower DR. Selection-based design of in silico dengue epitope ensemble vaccines. Chem Biol Drug Des. 2019;93(1):21-8. https:// doi.org/10.1111/cbdd.13357 Epub 12018 Nov 13325.

29. Reche PA, Keskin DB, Hussey RE, Ancuta P, Gabuzda D, Reinherz EL. Elicitation from virus-naive individuals of cytotoxic T lymphocytes directed against conserved HIV-1 epitopes. Med Immunol. 2006;5:1.

30. Shah P, Mistry J, Reche PA, Gatherer D, Flower DR. In silico design of Mycobacterium tuberculosis epitope ensemble vaccines. Mol Immunol. 2018; 97:56-62.

31. Sheikh QM, Gatherer D, Reche PA, Flower DR. Towards the knowledgebased design of universal influenza epitope ensemble vaccines. Bioinformatics. 2016;32(21):3233-9. https://doi.org/10.1093/bioinformatics/ btw3399 Epub 2016 Jul 3210.

32. Pass RF, Zhang C, Evans A, Simpson T, Andrews W, Huang ML, Corey L, Hill J, Davis E, Flanigan C, et al. Vaccine prevention of maternal cytomegalovirus infection. N Engl J Med. 2009;360(12):1191-9. https://doi.org/10.1056/ NEJMoa0804749.

33. Sabbaj S, Pass RF, Goepfert PA, Pichon S. Glycoprotein B vaccine is capable of boosting both antibody and CD4 T-cell responses to cytomegalovirus in chronically infected women. J Infect Dis. 2011;203(11):1534-41. https://doi. org/10.1093/infdis/jir1138.

34. Zhong J, Rist M, Cooper L, Smith C, Khanna R. Induction of pluripotent protective immunity following immunisation with a chimeric vaccine against human cytomegalovirus. PLoS One. 2008;3(9):e3256.

35. Angeletti D, Yewdell JW. Is It Possible to Develop a "Universal" Influenza Virus Vaccine? Outflanking Antibody Immunodominance on the Road to Universal Influenza Vaccination. Cold Spring Harb Perspect Biol. 2018;10(7). https://doi.org/10.1101/cshperspect.a028852 cshperspect.a028852.

36. Zhong W, Reche PA, Lai CC, Reinhold B, Reinherz EL. Genome-wide characterization of a viral cytotoxic T lymphocyte epitope repertoire. J Biol Chem. 2003;278(46):45135-44.

37. Molero-Abraham M, Lafuente EM, Reche P. Customized predictions of peptide-MHC binding and T-cell epitopes using EPIMHC. Methods Mol Biol. 2014;1184:319-32. https://doi.org/10.1007/978-1-4939-1115-8_18.

38. Vita R, Overton JA, Greenbaum JA, Ponomarenko J, Clark JD, Cantrell JR, Wheeler DK, Gabbard JL, Hix D, Sette A, et al. The immune epitope database (IEDB) 3.0. Nucleic Acids Res. 2015;43(Database issue):D405-12. https://doi.org/10.1093/nar/gku1938 Epub 2014 Oct 1099

39. Molero-Abraham M, Glutting JP, Flower DR, Lafuente EM, Reche PA. EPIPOX: Immunoinformatic Characterization of the Shared T-Cell Epitome between Variola Virus and Related Pathogenic Orthopoxviruses. J Immunol Res. 2015; 2015:738020. https://doi.org/10.1155/2015/738020 Epub 732015 Oct 738028.

40. Toseland CP, Clayton DJ, McSparron H, Hemsley SL, Blythe MJ, Paine K, Doytchinova IA, Guan P, Hattotuwagama CK, Flower DR. AntiJen: a quantitative immunology database integrating functional, thermodynamic kinetic, biophysical, and cellular data. Immunome Res. 2005;1(1):4. https:// doi.org/10.1186/1745-7580-1181-1184

41. Browne EP, Shenk T. Human cytomegalovirus UL83-coded pp65 virion protein inhibits antiviral gene expression in infected cells. Proc Natl Acad Sci U S A. 2003;100(20):11439-44.

42. Hensel G, Meyer H, Gartner S, Brand G, Kern HF. Nuclear localization of the human cytomegalovirus tegument protein pp150 (ppUL32). J Gen Virol. 1995:76(Pt 7):1591-601. https://doi.org/10.1099/0022-1317-76-7-1591.

43. Ciferri C, Chandramouli S, Donnarumma D, Nikitin PA, Cianfrocco MA, Gerrein R, Feire AL, Barnett SW. Structural and biochemical studies of HCMV $\mathrm{gH} / \mathrm{gL} / \mathrm{gO}$ and pentamer reveal mutually exclusive cell entry complexes. Proc Natl Acad Sci U S A. 2014;6:1767-72.

44. Fridkis-Hareli M, Reche PA, Reinherz EL. Peptide variants of viral CTL epitopes mediate positive selection and emigration of Ag-specific thymocytes in vivo. J Immunol. 2004;173(2):1140-50. https://doi.org/10.404 9/jimmunol.173.2.1140.

45. Alving CR, Koulchin V, Glenn GM, Rao M. Liposomes as carriers of peptide antigens: induction of antibodies and cytotoxic T lymphocytes to conjugated and unconjugated peptides. Immunol Rev. 1995;145:5-31.

46. Fleri W, Paul S, Dhanda SK, Mahajan S, Xu X, Peters B, Sette A. The immune epitope database and analysis resource in epitope discovery and synthetuc vaccine design. Front Immunol. 2017;8:278.

47. Li W, Godzik A. Cd-hit: a fast program for clustering and comparing large sets of protein or nucleotide sequences. Bioinformatics. 2006;22(13):1658-9.

48. Federhen S. Type material in the NCBI taxonomy database. Nucleic Acids Res. 2015;43(Database issue):D1086-98.
49. Edgar RC. MUSCLE: multiple sequence alignment with high accuracy and high throughput. Nucleic Acids Res. 2004:32(5):1792-7.

50. Garcia-Boronat M, Diez-Rivero CM, Reinherz EL, Reche PA. PVS: a web server for protein sequence variability analysis tuned to facilitate conserved epitope discovery. Nucleic Acids Res. 2008;36(Web Server issue):W35-41. https://doi.org/10.1093/nar/gkn1211 Epub 2008 Apr 1027.

51. Reche PA, Glutting JP, Reinherz EL. Prediction of MHC class I binding peptides using profile motifs. Hum Immunol. 2002;63(9):701-9.

52. Reche PA, Reinherz EL. Prediction of peptide-MHC binding using profiles. Methods Mol Biol. 2007;409:185-200.

53. Lafuente EM, Reche PA. Prediction of MHC-peptide binding: a systematic and comprehensive overview. Curr Pharm Des. 2009;15(28):3209-20.

54. Greenbaum J, Sidney J, Chung J, Brander C, Peters B, Sette A. Functional classification of class II human leukocyte antigen (HLA) molecules reveals seven different supertypes and a surprising degree of repertoire sharing across supertypes. Immunogenetics. 2011;63(6):325-35. https://doi.org/10.1 007/s00251-011-0513-0 Epub 02011 Feb 00259.

55. Nielsen M, Lundegaard C, Blicher T, Peters B, Sette A, Justesen S, Buus S, Lund O. Quantitative predictions of peptide binding to any HLA-DR molecule of known sequence: NetMHCIlpan. PLoS Comput Biol. 2008;4(7): e1000107. https://doi.org/10.1371/journal.pcbi.1000107.

56. Bui HH, Sidney J, Dinh K, Southwood S, Newman MJ, Sette A. Predicting population coverage of T-cell epitope-based diagnostics and vaccines. BMC Bioinformatics. 2006;7:153.

57. Hubbard SJ, Thornton JM. NACCESS, Computer Program. London: Department of Biochemistry and Molecular Biology, University College London; 1993.

58. Klausen MS, Jespersen MC, Nielsen H, Jensen KK, Jurtz VI, Sonderby CK, Sommer MOA, Winther O, Nielsen M, Petersen B, et al. NetSurfP-2.0: Improved prediction of protein structural features by integrated deep learning. Proteins. 2019;20(10):25674.

59. Schlessinger A, Yachdav G, Rost B. PROFbval: predict flexible and rigid residues in proteins. Bioinformatics. 2006;22(7):891-3. https://doi.org/10. 093/bioinformatics/btl032 Epub 2006 Feb 1092.

60. Altschul SF, Madden TL, Schaffer AA, Zhang J, Zhang Z, Miller W, Lipman DJ. Gapped BLAST and PSI-BLAST: a new generation of protein database search programs. Nucleic Acids Res. 1997;25(17):3389-402.

\section{Publisher's Note}

Springer Nature remains neutral with regard to jurisdictional claims in published maps and institutional affiliations.

Ready to submit your research? Choose BMC and benefit from:

- fast, convenient online submission

- thorough peer review by experienced researchers in your field

- rapid publication on acceptance

- support for research data, including large and complex data types

- gold Open Access which fosters wider collaboration and increased citations

- maximum visibility for your research: over $100 \mathrm{M}$ website views per year

At $\mathrm{BMC}$, research is always in progress.

Learn more biomedcentral.com/submissions 